\title{
Role of E-Health and Mobile Health in Management of Asthma: A Review Study
}

\author{
B Honarvar ${ }^{1,} ;$; K.B lankarani ${ }^{2}$ \\ ${ }^{1}$ Associate Professor, Health Policy Research Center, Institute of Health, Shiraz University of Medical Sciences, Shiraz, Iran \\ ${ }^{2}$ Professor, Health Policy Research Center, Institute of Health, Shiraz University of Medical Sciences, Shiraz, Iran \\ ${ }^{*}$ Corresponding author: B Honarvar, Assistant Professor of Community Medicine, Health Policy Research Center, Institute of Health, Shiraz University of Medical Sciences, Shiraz, P.O. \\ Box: 71348-45794, Iran. Tel: +98-7132309615, Fax:+98-7132309615, E-mail: honarvarbh32@yahoo.com
}

Received: 11 Dec 2016

Accepted: 01 Jan 2017

Epub: 23 Feb 2017

Ppub: 15 Jan 2018

\begin{abstract}
Background:Asthma is one of the common non-communicable diseases. Approximately 235 million people suffer from asthma around the world. Appropriate management of asthma can enable people to enjoy a good quality of life. The major international clinical guidelines now recommend including a self-management program in the routine management of patients with asthma. However, the implementation of self- management program in clinical practice, and their uptake by patients, is still poor.

Objectives: We investigated the literature for finding last evidences regarding the effect of e health and $\mathrm{m}$ health on different aspects of asthma management.

Methods: Academic databases of PubMed, Cochrane (central), and Scopus were searched, using different combinations of terms

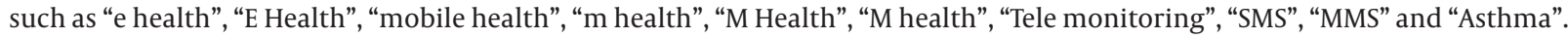
The searching was limited to the English language and only systematic review or meta-analysis studies were reviewed.

Results: We found 10 systematic review (SR) studies that included assessment of e health or $m$ health on management of Asthma. These SR studies included RCT (randomized control trials), before-after, and observational studies. These studies consisted of at least a total 134556 participants comprising of 10374 children. These studies were conducted in USA, Cameron, Italy, France, Canada, and some other countries. In these studies, different aspects of Asthma were evaluated such as self-management, medical adherence, scheduling visiting time by physicians, peak expiratory flow measurement, asynchronous communication and discussing between patients and providers, as well as monitoring 4 items of asthma (cough, night symptoms, sleep quality, and maximum tolerated activity). However, few studies investigated the cost effectiveness and long term efficacy of $m$ health or other e health facilities on management of asthmatic patients.

Conclusion:Although there are some strong evidences regarding the value of integrating $\mathrm{m}$ health in management of asthma, evidences about its long term benefit, cost-effectiveness, feasibility, and changing the outcome are limited. Therefore, the lack of large scale and strong clinical trial studies, for answering these questions is felt more than before.

Keywords: E-Health; M Health; Smart Phone; Asthma; Self-Management
\end{abstract}

